\title{
Growth zone segmentation in the milkweed bug Oncopeltus fasciatus sheds light on the evolution of insect segmentation
}

\author{
Tzach Auman(1) and Ariel D. Chipman* (1)
}

\begin{abstract}
Background: One of the best studied developmental processes is the Drosophila segmentation cascade. However, this cascade is generally considered to be highly derived and unusual, with segments being patterned simultaneously, rather than the ancestral sequential segmentation mode. We present a detailed analysis of the segmentation cascade of the milkweed bug Oncopletus fasciatus, an insect with a more primitive segmentation mode, as a comparison to Drosophila, with the aim of reconstructing the evolution of insect segmentation modes.

Results: We document the expression of 12 genes, representing different phases in the segmentation process. Using double staining we reconstruct the spatio-temporal relationships among these genes. We then show knock-down phenotypes of representative genes in order to uncover their roles and position in the cascade.

Conclusions: We conclude that sequential segmentation in the Oncopeltus germband includes three slightly overlapping phases: Primary pair-rule genes generate the first segmental gene expression in the anterior growth zone. This pattern is carried anteriorly by a series of secondary pair-rule genes, expressed in the transition between the growth zone and the segmented germband. Segment polarity genes are expressed in the segmented germband with conserved relationships. Unlike most holometabolous insects, this process generates a single-segment periodicity, and does not have a double-segment pattern at any stage. We suggest that the evolutionary transition to double-segment patterning lies in mutually exclusive expression patterns of secondary pair-rule genes. The fact that many aspects of the putative Oncopeltus segmentation network are similar to those of Drosophila, is consistent with a simple transition between sequential and simultaneous segmentation.
\end{abstract}

Keywords: Evo-devo, Arthropod, Body plan, Segment

\section{Background}

A defining feature of the arthropod body plan is its segmental organization. The segments - repeating morphological units along the anterior-posterior axis - are formed in a process known as segmentation. The formation of segments occurs very differently in different groups of arthropods. While there is no doubt that segments are homologous among all arthropods, when looking across their full phylogenetic spread, there is relatively little in common in the segmentation process. Nonetheless, from

\footnotetext{
* Correspondence: ariel.chipman@huji.ac.il

The Department of Ecology, Evolution \& Behavior, The Hebrew University of Jerusalem, Edmond J. Safra Campus, Givat Ram, 91904 Jerusalem, Israel
}

fruit flies to spiders to centipedes, segments are established utilizing a conserved set of transcription factors and signaling pathways, albeit, in different embryonic and cellular environments [1]. Mapping gene-expression patterns during segmentation, in organisms representing key points in the phylogeny and evolution of arthropods, enables the identification of conservation and divergence in the roles of relevant genes, and enables insights into the interplay between them, their functions in segmentation, and the way they have evolved to enable the different observed mechanisms of segmentation.

The segmentation process has been best studied in the fruit fly Drosophila melanogaster [2]. Drosophila

(c) The Author(s). 2018 Open Access This article is distributed under the terms of the Creative Commons Attribution 4.0 International License (http://creativecommons.org/licenses/by/4.0/), which permits unrestricted use, distribution, and reproduction in any medium, provided you give appropriate credit to the original author(s) and the source, provide a link to the Creative Commons license, and indicate if changes were made. The Creative Commons Public Domain Dedication waiver (http://creativecommons.org/publicdomain/zero/1.0/) applies to the data made available in this article, unless otherwise stated. 
segmentation is the text-book example of a simple embryological patterning system and is taught in virtually every developmental biology course worldwide. Despite its canonical status in developmental biology, it has been known for over two decades that the Drosophila pathway is unusual among arthropods and is highly derived [1]. It is therefore extremely interesting to understand how this derived process evolved from the ancestral arthropod mode.

Segmentation in Drosophila is more or less simultaneous, and is effected through a series of tiered sets of genes, dividing the embryo into smaller and smaller units, culminating in a set of genes expressed in every segment $[2,3]$. This process and the genes involved therein are usually referred to as the "segmentation cascade". In contrast, the segmentation process in many arthropods is sequential, with segments being formed one or two at a time, from a posterior growth zone (also known as the "segment addition zone"). Stahi and Chipman [4], traced the evolution of these two modes of segmentation across insects, and showed a complex evolutionary history, including intermediate forms between the two, and cases of parallel gains and losses of both. They suggested that the roots of the Drosophila segmentation cascade appear early in evolution, before the radiation of holometabolous insects (those insects with a bi-phasic life cycle punctuated by dramatic metamorphosis). Their interpretation is consistent with an idea originally put forward by Peel [5], according to which there was a gradual transition of control over segmentation from an ancestral posterior cycling mechanism to a gap-gene based simultaneous patterning mode.

The frequent switches between sequential and simultaneous segmentation, and the existence of numerous intermediate states indicate that the short-germ/long-germ dichotomy is not real, and that despite differences in the context and dynamics of the different segmentation modes, they must have many fundamental commonalities. This view is corroborated by recent detailed analyses of the Drosophila segmentation cascade that indicate that not only are the early-acting gap genes dynamic in space and time [6, 7], these dynamics are necessary for the correct activity and positioning of the later acting pair-rule genes, which directly define segmental position [8]. Modeling the activity of the pair-rule gene network has shown that minor changes in the gap-gene input can lead to a transition between short-germ and long-germ development [8]. A separate modeling approach has also shown that differences in the gradient of posterior signaling molecules can also lead to easy switching between the two modes [9]. With these new theoretical insights, we set out to increase the extent of experimental data regarding the diversity of gene function in the different modes in order to shed more light on the evolution of insect segmentation.
Our model organism of choice is the milkweed bug Oncopeltus fasciatus [10]. Oncopeltus is a member of Paraneoptera, which is the sister group to Holometabola. As such, it is ideally situated as an outgroup to the hyper-diverse and widely studied holometabolous insects, and can serve to polarize changes in the segmentation program in a comparison between the two most widely studied insects: Drosophila and the flour beetle Tribolium castaneum, as well as other holometabolans. Previous work on Oncopeltus has also shown that it tends to be fairly conservative, and represents many ancestral characteristics in its developmental program [10]. The anterior segments in Oncopeltus are patterned simultaneously, through a process that bears many similarities to the Drosophila cascade. Posterior segments are patterned sequentially from a growth zone. Our previous work on the Oncopeltus growth zone [11] showed that it is divided into two functional domains: a posterior growth zone with high levels of cell proliferation and stable gene expression patterns, and an anterior growth zone with dynamic gene expression patterns and a reduced level of cell proliferation. Comparing this organization to that found in other arthropods, we suggested that it is a general feature of sequentially segmenting arthropods.

Our previous work analyzed only a small number of genes in the segmenting growth zone of Oncopeltus. This sample allowed us to demonstrate that segments are formed one at a time, unlike the two-segment periodicity found in both Drosophila and Tribolium (and convergently in geophilomorph centipedes [12]). Indeed, the ortholog of the Drosophila pair-rule gene evenskipped (eve), famously expressed in a two-segment periodicity in the Drosophila blastoderm [13], is expressed in every segment in Oncopeltus.

In the current work, we have looked at orthologs of several more genes involved in the Drosophila segmentation cascade, including most pair-rule genes and segmentpolarity genes (the gap genes have been studied in detail previously [14-17]). We have focused on sequential segmentation in the abdomen during the germband stage, taking advantage of the fact that in species with terminal growth, the anterior-posterior axis serves as a proxy for a time axis, with more anterior regions representing later stages in the process of growth and segmentation [11]. This allows us to identify the temporal sequence of gene activation and to extrapolate to the sequence of developmental events involved in generating segments. Adding RNAi mediated knock-down of some of these genes gives additional information about their function. Placing this in the comparative context described above allows us to discuss some of the key steps in the evolution of the segmentation process in insects. 


\section{Results}

Expression patterns of "segmentation cascade" genes We followed the expression patterns of 12 genes, mostly orthologues of the pair-rule and segment-polarity genes, during the formation of the abdominal segments of Oncopeltus fasciatus. Some of the genes discussed herein (eve, $D l$, cad, inv) have previously been described and will only be mentioned briefly, adding details that have not been reported before. We present them roughly in the order of their appearance, from posterior to anterior. To facilitate inter-species comparison, we only report on genes that are orthologs of genes involved in the segmentation cascade of Drosophila, realizing that this does not give the full picture, as there may be other genes involved in segmentation in Oncopeltus that do not have such a role in Drosophila $[18,19]$. Note that we use the terms pair-rule genes and segment-polarity genes as convenient shorthand for orthologs of genes that have a pair-rule / segment-polarity role in Drosophila, and this does not a-priori imply a similar role in Oncopeltus.

\section{Even-skipped (eve)}

The expression pattern of eve $[11,20]$ includes a domain of solid expression in the posterior growth zone, and a striped expression domain in the anterior growth zone (Fig. 1a-a'). The number of eve stripes early in abdominal segmentation can be as high as five or six, while by the end of the segmentation process, there is only a single eve stripe anterior to the solid expression domain. In some stained embryos, the posterior-most eve stripe is in contact with the solid expression domain in its medial portion, giving the impression of a stripe "peeling off" from the solid domain [20].

\section{Odd-skipped (odd) and sister of odd-and bowel (sob)}

The expression patterns of odd (Fig. 1b-b') and its paralog sob (Fig. 1c-c') are nearly identical to each other, and both are remarkably similar to that of eve. They also have a solid expression domain in the posterior growth zone, and a striped expression in the anterior growth zone. However, unlike eve, the expression of odd and sob in the posterior growth zone is graded, with highest expression in the anterior margin of the posterior growth zone, tapering off posteriorly, and ending before the posteriormost end of the embryo. The striped expression of odd and sob extends into the segmented germband slightly more than that of eve.

\section{Runt (run)}

Like odd and eve, run (Fig. 1d-d') is defined as a "primary pair-rule gene" in Drosophila. In Tribolium, these three genes were found to work together in a pair rule regulatory circuit generating the repeating pattern of the segmentation process [21]. The probe for run gave very weak signal in our hands, so we could not analyze it at the level of detail we could for other genes. In the Oncopeltus germband, run does not display a striped expression pattern in the growth zone or in the segmented germband, in contrast with all other "segmentation cascade" genes in this study. It is mainly expressed in two to three broad graduated domains within the growth zone. This pattern is highly dynamic and variable among embryos, but we were unable to correlate this dynamic activity with that of the other genes we have looked at. In addition to its expression in the growth zone, in late stages of segmentation, run is expressed in paired domains in the germband near the ventral midline, probably representing neural precursor tissue. The aforementioned four genes are the only pair-rule gene orthologs that are expressed in the posterior growth zone.

\section{Odd-paired (opa) and sloppy-paired (s/p)}

These two genes are often defined as "secondary pair-rule genes" in Drosophila. In the Oncopeltus anterior growth zone, opa is expressed in a striped pattern (Fig. 2a-a'), resembling that of eve, odd and sob. Unlike these genes, opa is not expressed in the posterior growth zone at any stage. The number of stripes in the growth zone varies from 2 to 3 stripes early in the segmentation process to a single stripe at later stages. These stripes are more anteriorly located than the eve and odd/sob stripes. Expression of opa continues into the segmented germband and expression is maintained in narrow stripes in the posterior of each segment throughout the germband stage.

The expression of $s l p$ (Fig. 2b-b') is similar to that of opa with two main differences: the expression stripes are broader in the germband and are found in a more anterior-medial position in each segment. A more subtle distinction is that slp has a weak posterior-anterior expression gradient in each stripe, both in the anterior growth zone and in the germband.

\section{Hairy (h)}

Expression of $h$ (Fig. 2c-c') is weakly noticeable in the posterior growth zone of early germband embryos. In the anterior growth zone, it is expressed in two faint stripes, and in a narrow stripe in the posterior of every mature segment. Like run, it also shows expression in the mesodermal cells of the growth zone. Segmental expression fades in mature segments later in development.

\section{Hedgehog (hh)}

Known from Drosophila as a segment polarity gene, $h h$ is expressed not only in every germband segment, but also in stripes in the anterior growth zone, similar to the pair-rule gene orthologs (Fig. 3a-a'). It is visible in the posterior region of the anterior growth zone as a wide stripe, not fully resolved and not always clearly separated 

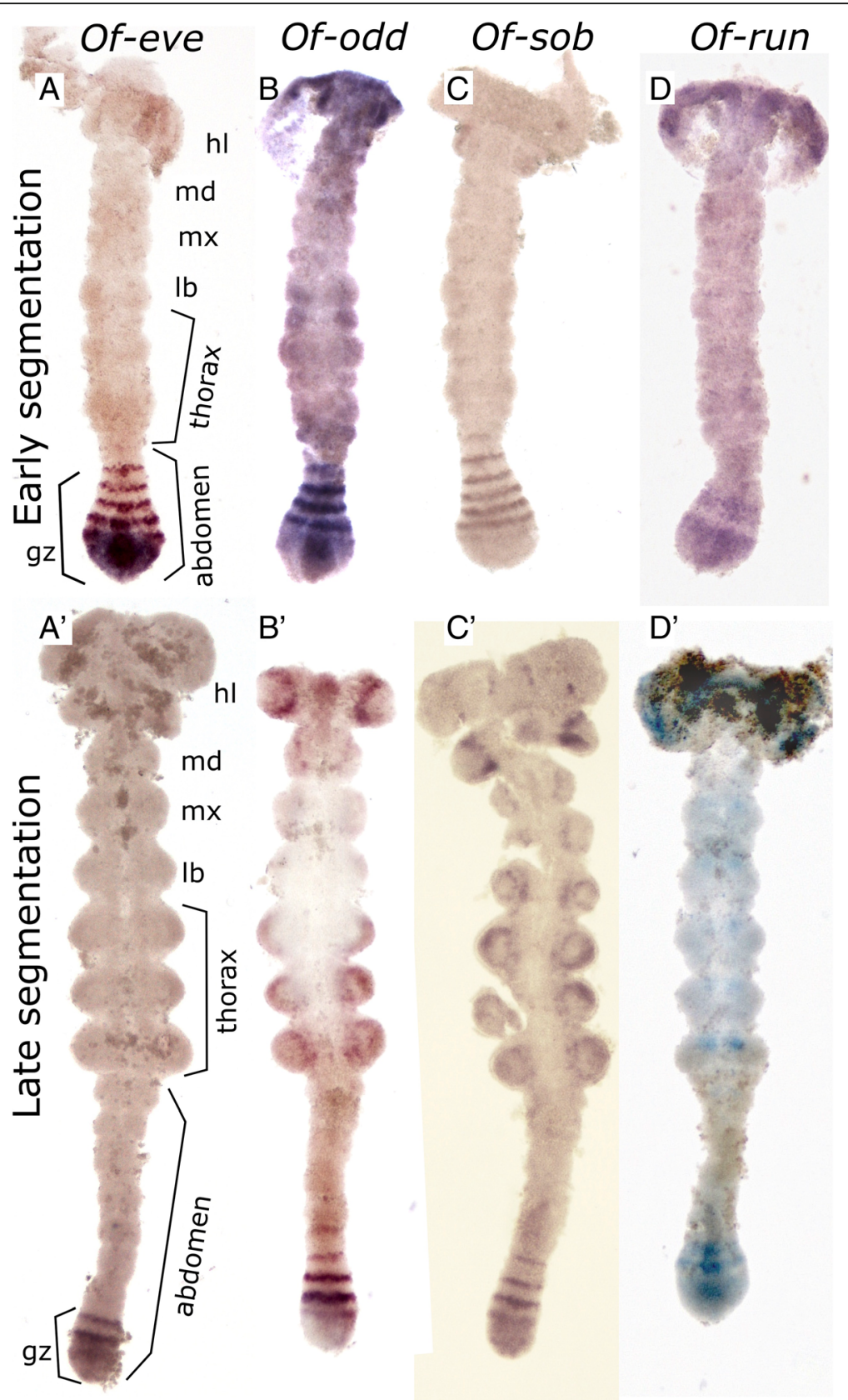

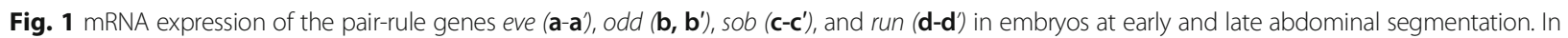
the early segmenting germband (44-46 h after egg laying (hAEL)), eve (a), odd (b) and sob (c) all display a similar expression pattern in the anterior growth zone (GZ) composed of 4-6 stripes, corresponding to nascent segments. The main difference between the expression pattern of these genes is most notable in the posterior GZ where eve is steadily expressed, whereas odd and sob show weaker and graduated expression. Run (d-d') expression is very different form the other three genes. It is expressed in two broad stripes corresponding to the anterior and posterior $\mathrm{GZ}$, and in patches in the anterior thoracic and gnathal segments. In late germband stages (50-52 hAEL) (a'- d') we see striped pattern of eve, odd and sob maintained, but with a smaller number of stripes. Expression of run is decreased to a single broad band in the anterior GZ. In addition, odd, sob and run are expressed in the limb buds. Embryos were chosen from within the aforementioned age range to be as similar as possible in developmental stage, based on size and shape of the germband and growth zone. In all images anterior is to the top. Abbreviations: gz, growth zone; hl, head lobe; md, mandibular segment; mx, maxillary segment; Ib., labial segment

from the next anterior, better defined stripe. The third $h h$ stripe is fully separated from the two prior stripes, as are the more anterior stripes. The segmental stripes are situated in the posterior of each segment. In addition to the striped expression pattern, $h h$ is expressed in a single patch at the very posterior of the embryo. 


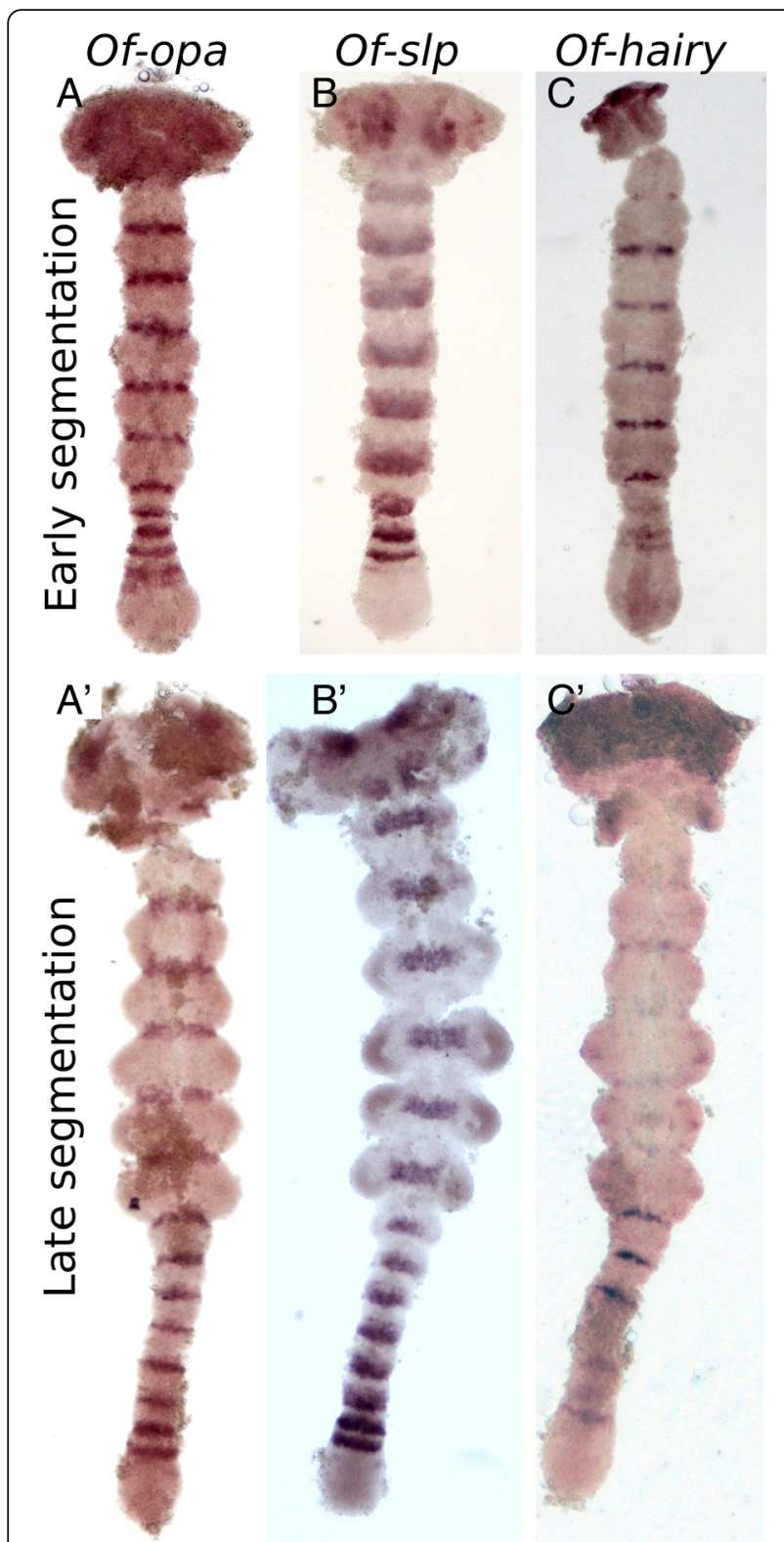

Fig. 2 Expression of the pair rule genes opa (a-a'), s/p (b-b') and $h$ (c-c') in embryos at early and late abdominal segmentation.

Throughout development, opa $\left(\mathbf{a}, \mathbf{a}^{\prime}\right)$ is expressed in a narrow band at the border of every segment but is not found in the posterior GZ. $\operatorname{slp}\left(\mathbf{b}, \mathbf{b}^{\prime}\right)$ is more broadly and anteriorly expressed in each segment. The earliest, most posterior stripes are thin, and increase in breadth anteriorly. In later stages (b'), it shows diffuse expression in the limb buds. $h$ expression $\left(\mathbf{c}^{-} \mathbf{c}^{\prime}\right)$ is similar to that of opa in nascent segments but is weaker in mature segments. In the anterior $G Z$ it is expressed in two stripes at the anterior of the anterior GZ, and more weakly in the posterior GZ. There is also weak punctate expression in the limb buds. Embryo ages are as in Fig. 1. In all images, anterior is to the top

\section{Wingless (wg) and invected (inv)}

We have previously described the expression of $w g$ in the Oncopeltus blastoderm [4], but not in the germband. Expression of the segment polarity gene $w g$ begins in the

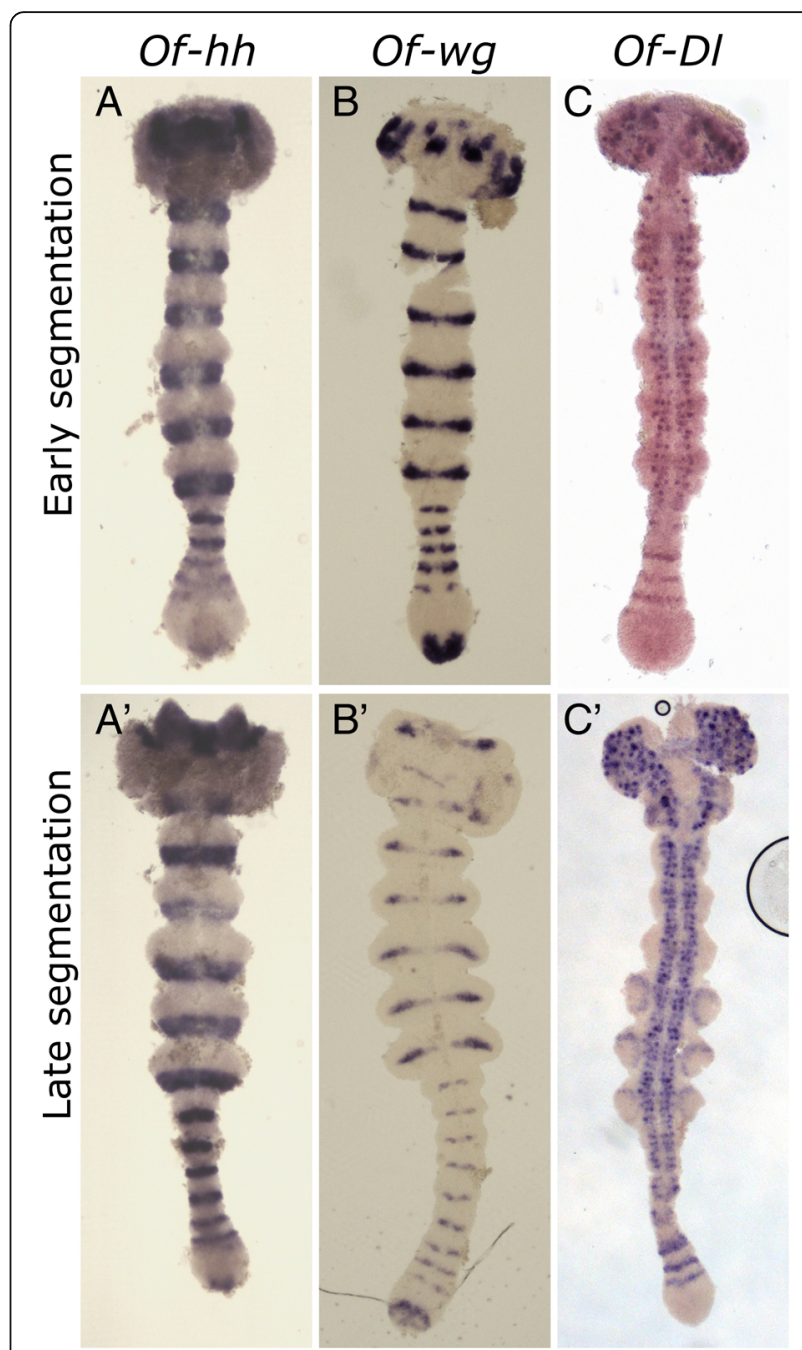

Fig. 3 Expression of the segment polarity genes $h h\left(\mathbf{a}-\mathbf{a}^{\prime}\right)$, and wg $\left(\mathbf{b}^{-} \mathbf{b}^{\prime}\right)$, and the Notch ligand $D /(\mathbf{c}-\mathbf{c})^{\prime}$. For the most part $h h$ expression corresponds to that of inv/en, defining the posterior of each segment. Unlike inv/en, $h$ h is expressed in the anterior GZ, and in a patch at the posterior GZ. wg ( $\mathbf{b}$, $\left.\mathbf{b}^{\prime}\right)$ is expressed in the middle of each segment. Like $h h$, it is expressed in a patch in the posterior of the GZ. DI (c-c') is found to be expressed in a series of stripes in the anterior GZ, and in a punctate pattern in neuronal tissue.

Embryo ages are as in Fig. 1. In all images, anterior is to the top

forming segment, initially as two lateral dots, later expanding and fusing to form a segmental stripe in the middle of each segment (Fig. 3b-b'). The segmental stripes are notably thinner medially. In addition to its segment-polarity pattern, $w g$ is strongly expressed in the posterior growth zone. In the early stages of the germband it appears in the posterior pole of the embryo, and as segmentation progresses, it gains a crescent like shape beginning at the medial part of the posterior growth zone, curving anteriorly. At later stages, expression moves slightly anteriorly and gains an $M$ shape. Expression of inv (an engrailed paralog) has been described in 
several previous publications. It is expressed in the posterior of every segment in the germband.

\section{Caudal (cad) and Delta (DI)}

We have previously described the expression of $D l$ and cad in the Oncopeltus germband [11]. Briefly $-D l$ Expression is seen in the anterior growth zone in 2-3 stripes, and in a punctate neural system related expression pattern in the segmented germband (Fig. 3c-c'). Expression of cad is restricted to the posterior growth zone through the germband stage.

\section{Relative expression domains}

To clarify the spatial relationships among the different genes, we carried out a series of double stainings (Fig. 4). Although not all combinations worked, we have sufficient pair-wise comparisons to be able to reconstruct the relative position of all of the genes studied, with the exception of run (summarized in Fig. 5).

Four of the genes we studied are expressed in solid domains throughout the posterior growth zone - cad, eve and $o d d / s o b$. We carried out double staining for cad and eve (Fig. 4a) to see whether they share an anterior border in the posterior growth zone (the border between the anterior and posterior domains of the growth zone). We could detect no difference in the anterior extent of these genes, indicating a single uniform border within the growth zone.

The expression patterns of eve and odd are similar (Fig. 4b), With full overlap in the posterior growth zone. However, looking at their anterior expression shows that they overlap only partially, with eve expression being anterior to that of odd in any given stripe. We did not double-stain odd and sob, however, they both show the same relationship to eve (Fig. 4c) suggesting that their expression patterns fully overlap.

The two secondary pair-rule genes, opa and slp (Fig. $4 \mathrm{~d})$ are expressed in complementary patterns in the anterior growth zone, with slp forming the posteriormost stripe. As segmentation progresses, in the later stripes of the anterior growth zone, a gap appears anterior to the opa stripe and posterior to the slp one. In the segmented germband the stripes are fully separated and occupy distinct regions of the nascent segment. Comparing slp with eve (Fig. 4e) shows that they have a narrow domain in the anteriormost growth zone where both are expressed. Expression of $s l p$ appears as a faint narrow band in the lateral anterior growth zone, anterior and adjacent to the second eve stripe. The second $s l p$ stripe is already much stronger, but still shows a gap in the midline where eve is expressed, indicating that at this stage, these two genes are probably not co-expressed, but are both present in different areas of the same position along the anterior-posterior axis. The third slp stripe is completely resolved to the anterior of the final, most anterior eve stripe. As visible in the single stainings, $s l p$ is expressed in a graduated manner, strongly expressed in the posterior of the band, weakening towards the anterior but still with a well-defined anterior border, after which there is a gap where neither eve nor slp are expressed. The relative expression of opa and slp suggests that eve overlaps opa in the anterior of each stripe.

The expression of eve and inv (Fig. 4f) overlap exactly in the only region where they are co-expressed - the posteriormost segment (as previously shown by Liu and Kaufman [20]). The domains of eve and hh (Fig. 4g) also overlap, but this overlap extends through the entire anterior growth zone. Thus, we conclude that $i n v$ and $h h$ also overlap. The third segment polarity gene we have looked at, $w g$ abuts $h h$ and sits anterior to it (Fig. $4 \mathrm{~h}$ ). Thus, the expression of $h h$ can be seen as a combination of the posterior expression of eve and the anterior expression of inv.

Finally, the expression of $D l$ lies anterior to that of eve (Fig. 4i), perhaps with a slight overlap. Thus, when it is still expressed in stripes, $D l$ overlaps the expression domain of slp. The picture is completed by the anterior expression of $h$, which lies adjacent and posterior to inv, but is expressed earlier in any given segment (Fig. 4j).

\section{Spatial dynamics of the segmentation genes}

In order to gain a better understanding of the dynamic pattern of the segmentation genes over time, we have measured the expression levels of three representative genes, eve, odd and $h h$, along the anterior-posterior axis (Fig. 6a-c, a'-c'). We summed the pixel intensity for every point along the posterior-anterior axis on photographs of stained embryos. For $h h$ and eve, we followed this up in a large sample of $>50$ embryos, and plotted summed pixel intensity along the axis over developmental time on a three-dimensional graph (Fig. 6d-e. See methods), including relative developmental age (a value-less order based on germband length), position along the axis (using the posterior boundary of the third thoracic segment as the origin) and normalized expression level. The individual embryos used for this analysis covered a range of stages, but are distributed randomly across this range. The time axis is thus not linear, but rather a category axis of increasing size, which serves as a good proxy for developmental age. The plot for $h h$ (Fig. 6d), which is expressed in every segment starting from the anterior growth zone, shows that indeed size serves as a good proxy for age, since we can see the $h h$ segmental stripes appearing in correct sequence. These plots allow us to follow the development of the expression patterns of the two genes, and by extension, shed some light on the dynamics of the entire process.

Using this visualization, we show that when eve stripes first peel off from the posterior growth zone's solid expression domain (Fig. 6e), they remain stationary relative 

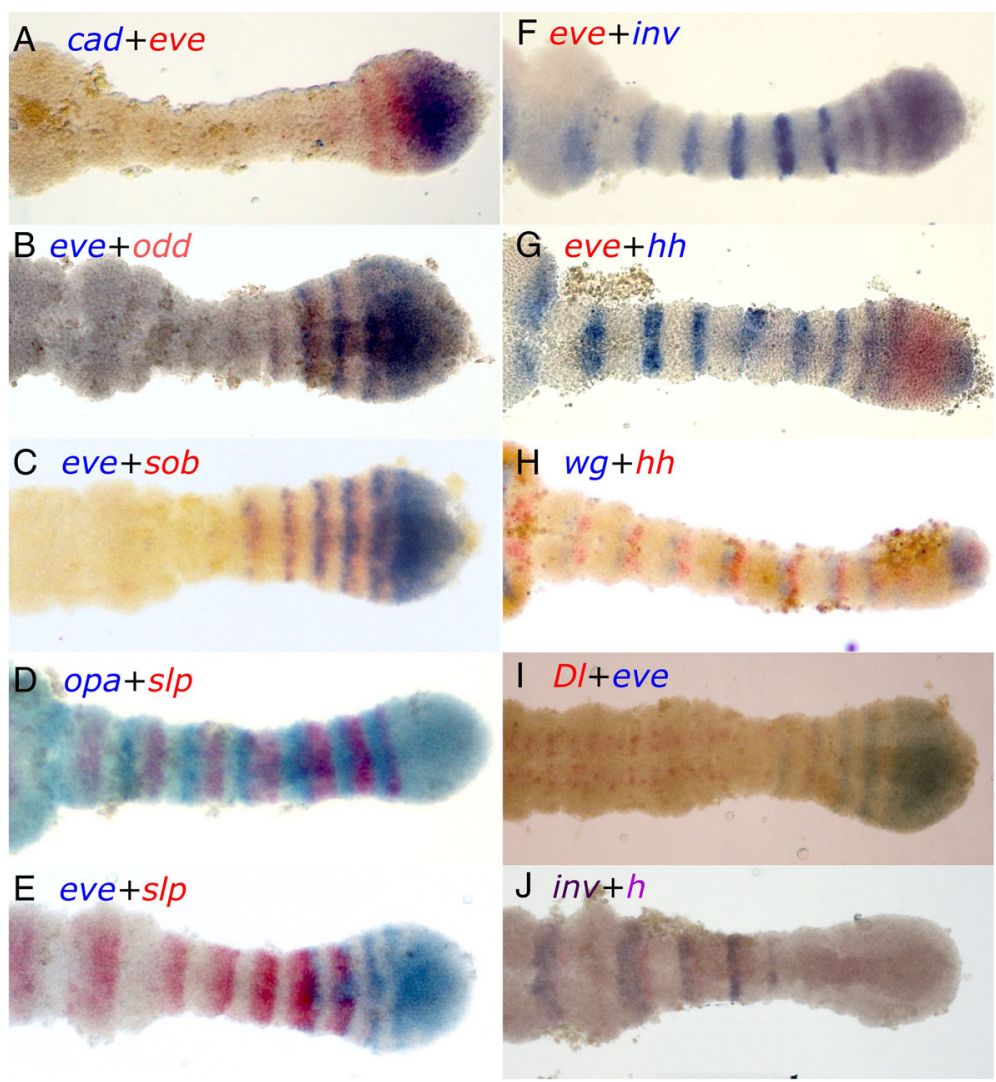

Fig. 4 Relative expression domains of different genes in the posterior of the embryo, as illustrated by combinations of double staining. a cad and eve are co-expressed in the posterior GZ, with eve stripes extending into the anterior GZ. $\mathbf{b}$ eve and odd, are shifted relative to each other, with overlapping expression in a narrow area (posterior of eve and anterior of odd), but with most of the expression separate. c The relationship between eve and sob is identical to that between eve and odd. $\mathbf{d}$ opa and slp are expressed in adjacent domains with no observable overlap. The posterior stripes are complementary and cover the entire anterior GZ. In later, more anterior stripes, as the segment grows, a region without opa or $s / p$ emerges, anterior to opa and posterior to $s / p$. In later stages, opa and s/p are completely separated. e s/p expression begins just as eve expression is fading. In the segments where they are both expressed, slp is expressed to the anterior of eve. $\mathbf{f}$ The transition between eve and inv defines the GZ-germband border. At the transition, they are co-expressed in one or two stripes, in which their domains overlap. $\mathbf{g}$ The first stripes of $h$ h expression overlap those of eve in the anterior GZ. $\mathbf{h} h \mathrm{~h}$ is immediately adjacent and posterior to $\mathbf{w g}$ in segmental stripes beginning in the anterior GZ. This relation is maintained in the posterior GZ, where both are expressed in non-overlapping patches. $\mathbf{i}$ D/ and eve are partially coexpressed in the anterior GZ, with $D /$ extending more anteriorly than eve, and eve beginning posteriorly to their overlapping domain. $\mathbf{j} h$ is expressed immediately posteriorly to inv, beginning slightly more anteriorly. Anterior is to the left in all images

\begin{tabular}{|c|c|c|c|c|}
\hline & Seg $n-1$ & $\operatorname{Seg} n$ & Anterior GZ & Posterior GZ \\
\hline \multicolumn{5}{|l|}{ cad } \\
\hline \multicolumn{5}{|c|}{ eve } \\
\hline \multicolumn{5}{|c|}{$d d / s o b$} \\
\hline \multicolumn{5}{|c|}{ run } \\
\hline hh & & & & \\
\hline \multicolumn{5}{|l|}{ sip } \\
\hline \multicolumn{5}{|l|}{ opa } \\
\hline \multirow{2}{*}{\multicolumn{5}{|c|}{$\frac{h}{i n v}$}} \\
\hline & & & & \\
\hline wg & 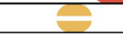 & $\because$ & & \\
\hline$D$ & & 8 & & \\
\hline
\end{tabular}

Fig. 5 Schematic representation of the relative expression patterns of all the genes discussed, as deduced from the double and single stainings. Question marks indicate cases with ambiguous staining, or where double staining was not possible, preventing us from identifying exact relative expression domains 


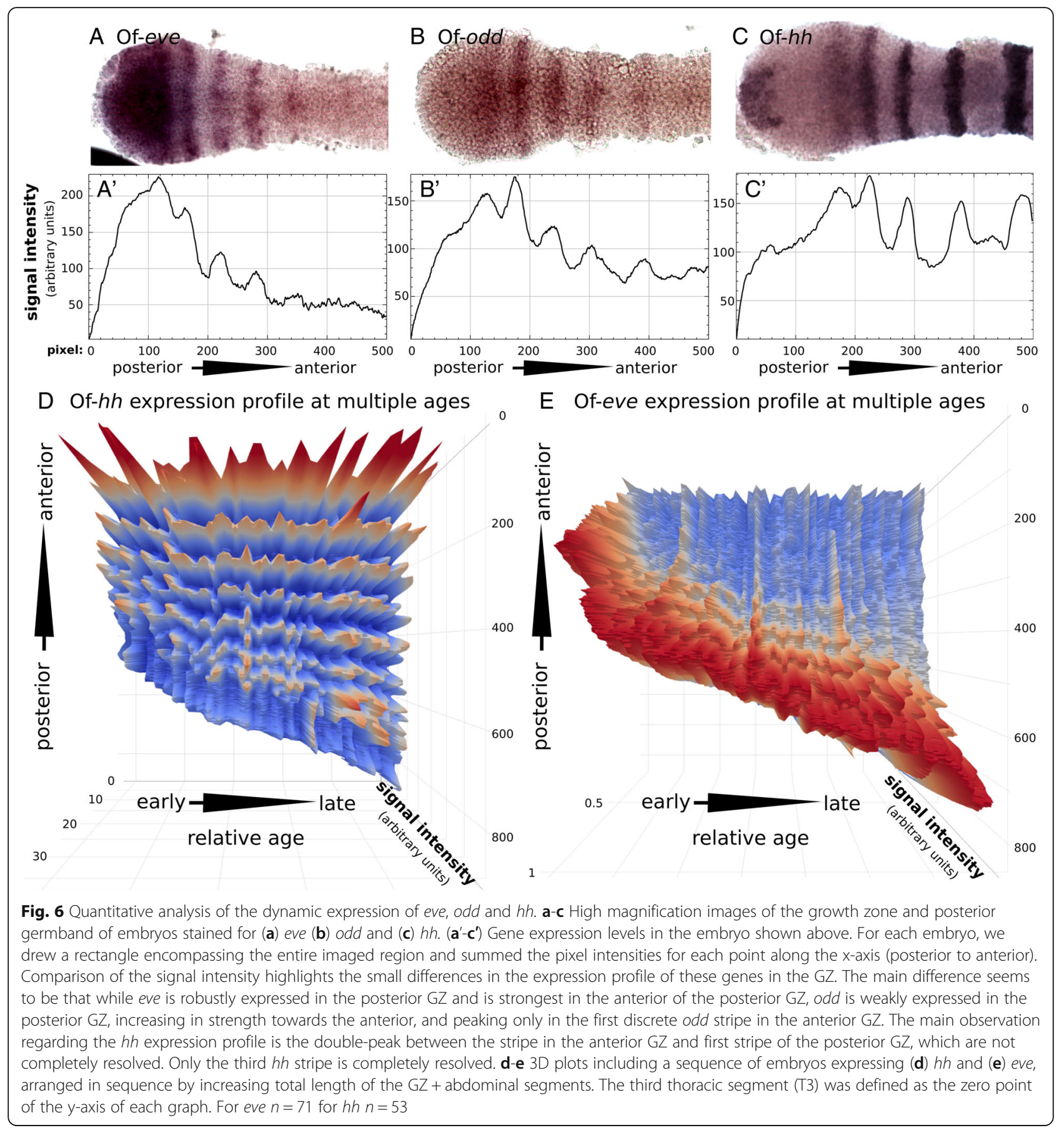

to the germband (represented by the third thoracic segment) but shift slightly in position relative to the solid expression domain of eve in the posterior growth zone. The stripes of $h$ h expression, in contrast, remain in a constant position relative to the third thoracic segment after they are formed.

\section{RNAi experiments}

Following the detailed analysis of gene expression patterns, we went on to examine the function of representative genes in the segmentation process by knocking them down through RNAi. For each gene knocked down, we collected early germband stages and late germband stages of RNAi embryos and stained them for inv and eve. In addition, we collected a small number of pre-hatching larvae to identify morphological phenotypes.

RNAi experiments have previously been conducted for some of our genes of interest. Knocking down eve leads to a truncation of the embryo and a complete loss of all growth zone derived segments [20]. Knocking down the 
segment polarity genes, inv (previously identified as engrailed) and wg leads to malformed segmental boundaries, but does not lead to any specific truncation phenotypes [22]. We have knocked down the second important primary pair rule gene odd, the secondary pair rule gene $s l p$, and the remaining segment polarity gene $h h$. Most of the eggs in these experiments were fixed during development, and only a small percentage were left to develop until hatching. Thus, we have a relatively small sample of larval phenotypes (Additional file 1). For all three genes, the larval phenotypes were fairly uniform and could not be broken down into relevant specific phenotypic classes. Thus, we treat them all together (but see difference between the two dsRNA fragments in $s l p$ below).

Early odd-RNAi germband embryos (Fig. 7b) exhibit a reduction in the distance between the anterior gnathal and thoracic segments, and a much broader expression of inv. The maxilla and labium are closer together with cells between them expressing inv ectopically. T1 and T2 are also wider and closer, and are fused in the midline. T3 seems to be normal, and so do the abdominal segments present at this stage. The growth zone exhibits no visible abnormalities.

In later, fully segmented germband embryos (Fig. 7b'), we see that this phenotype has progressed to limb fusion: $\mathrm{T} 1$ and $\mathrm{T} 2$ are fused. T3 remains mostly separated. In the abdominal segments, we see a similar effect of segment fusion and occasional ectopic expression of inv between segmental stripes. Notably, we see no evidence of segment deletion. The number and location of the segments is normal, but with defective segment boundaries. This is true both for the blastoderm derived anterior segments and for the growth zone derived posterior segments. The larval odd RNAi phenotypes are remarkably uniform, and nearly all show the same findings: segments are formed with irregular boundaries, appendages are fused and we see deformation in the head, mainly indicating abnormal midline closure. A very small number of hatchlings (around 10\%) show a stronger phenotype with anterior or posterior segments missing (Additional file 1).

Similarly, slp RNAi embryos retain the normal number of segments (Fig. 7c'), but these are misshapen. Already in the early germband embryo (Fig. 7c) the embryos are noticeable wider, and we can see an expansion and ectopic expression of inv in the midline of the blastoderm derived segments. At later stages, the ventral (medial) region is much wider and thinner, with unusual secretions obscuring the cells (possibly indicating apoptosis). Expression of inv is lost from the ventral portions of these segments (Fig. 7c'). The first abdominal inv stripes are expressed relatively normally, and only in the later embryo (Fig. 7c') do we see that the segment border is malformed in the mediolateral aspect of the abdomen, lacking normal expression of inv. The most striking outcome of $s l p$ RNAi is the loss of thoracic appendages. Some embryos are completely devoid of appendages, while some maintain residual stumps of limbs T1 and T2. This is also seen in the hatchlings (Fig. 7c"), where instead of limbs we find actual holes in the cuticle. In under $10 \%$ of the hatchlings, we found more severe phenotypes with possible failed dorsal closure. We repeated the RNAi experiment with a second fragment. The results were similar, but the phenotypes were generally weaker (Additional file 1), and we saw none of the extreme phenotypes. Interestingly, both in germband embryos and in hatchlings, we see that the T2 limb is lost before the other limbs.

RNAi for $h$ h (Fig. 7d-d") gave similar results to those previously reported for the other segment polarity genes [22]. All segments are present, and germband embryos look almost normal. However, hatchlings are compressed and show disrupted segmental boundaries. Malformations of the head are seen both in the germband embryos and in the hatchlings.

We looked at the expression of eve in RNAi embryos for all three genes. In all cases, the expression of eve in the growth zone is almost indistinguishable from wildtype expression (Additional file 2). However, in $h h$-RNAi embryos, we see ectopic expression of eve in a stripe in the head region, and in odd-RNAi embryos we see ectopic expression in the midline of the germband.

\section{Discussion}

\section{The segmentation "cascade" of Oncopeltus}

Our results, coupled with our previous analyses of segmentation in Oncopeltus [4, 11], allow us to reconstruct the series of molecular events involved in defining segments from the growth zone (Fig. 5). The fact that the first eve stripe is the most posterior segmental expression pattern, suggests that the first event in the segmentation process is the separation of an eve stripe from the posterior growth zone and a limited movement of eve expression across cells at the posterior margin of the anterior growth zone. We suggest that the first eve stripe activates the entire downstream sequence of expression patterns. However, we still do not know what generates the repeating process (or oscillator) that causes eve stripes to peel off and move anteriorly. The two possible candidates [23] are Delta-Notch signaling, as found in centipedes, spiders and branchiopod crustaceans [2427], or a pair-rule gene circuit, as found in Tribolium [21]. Neither one of these candidates is fully consistent with our data. $D l$ is not expressed at the right time and place to be upstream of eve, and knocking it down does not disrupt the early segmentation process, but rather the later stages of segmental boundary formation [4]. However, other unstudied Notch ligands could be involved in generating a repeated pattern. A gene circuit 

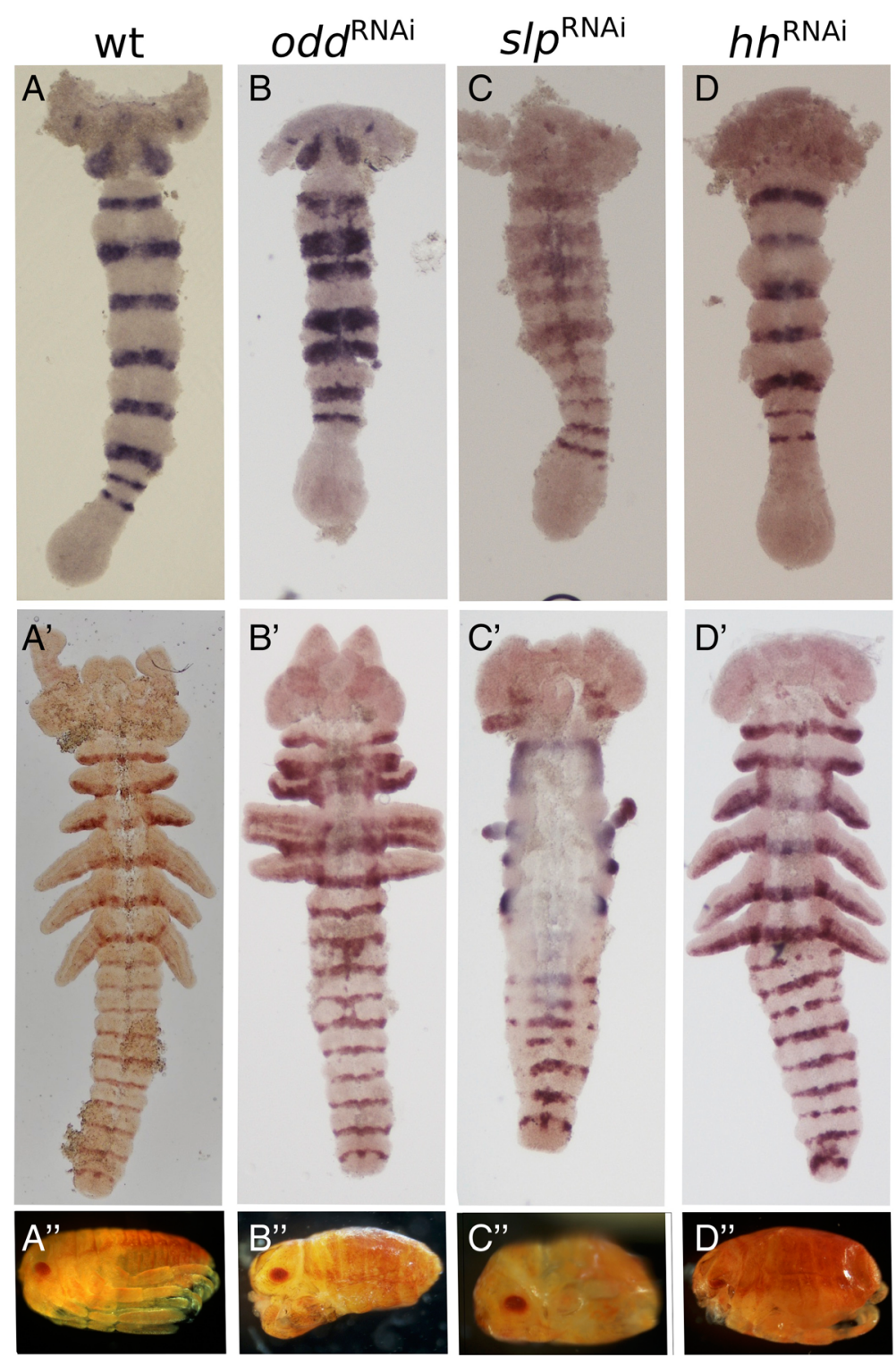

Fig. 7 Segmental phenotypes following knock-down of odd (b-b"), slp (c-c') and $h$ h (d-d"), in early and late germband embryos stained for inv and in hatchlings. a-a" Wildtype embryos and hatchling. $\mathbf{b}$ In the early germband embryo odd RNAi embryos mainly display widening of inv expression in the thoracic segments, and fusion of segments in the embryonic midline. $\mathbf{b}^{\prime}$ In later stages, appendages are fused, and the borders of some abdominal segments are also ill-defined, sporadically fused or narrowed. In both embryonic stages, slight ectopic expression of Of-inv is

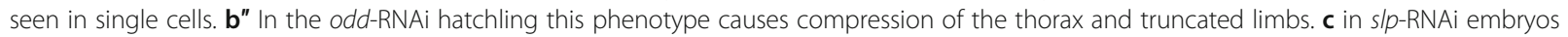
thoracic inv expression is broader in the early germband embryo, and abnormally expressed in the midline. $\mathbf{c}^{\prime}$ The later s/p-RNAi embryo displays severe truncation of all appendages, with only limb buds of T1 and T3 remaining. In addition, we see malformation of the abdominal segment boundaries, where gaps in inv expression can be seen. The s/p-RNAi embryo is also wider than WT embryos and has an apparent breakdown of midline tissues. c" The slp-RNAi hatchlings are compressed with almost no segmental boundaries, and holes appear in the lateral parts of the embryo, where the limbs are missing. $\mathbf{d}$ Early hh-RNAi embryos seem to be almost completely normal, only displaying some minor head aberration. $\mathbf{d}^{\prime}$ Aberrations of the head are also seen in the late germband embryos which seems to lack some folds and finer details of the head structure. Abdominal segment borders are also affected, containing gaps and ectopic expression of inv in sporadic cells. d" In hatchlings, the head is greatly reduced and malformed. Segmental borders can be seen, but they are disrupted. Limbs develop normally

as in Tribolium is a possibility, but not with the exact same interactions, since knocking down odd expression does not affect eve expression in the growth zone, and because odd and eve expression domains overlap, making a repressive interaction between them unlikely. We cannot draw clear conclusions about the possible role of run in such a circuit due to the poor quality of our current run staining, but the dynamic nature of the pattern suggests that there is room for a more in depth analysis of this gene. 
Following striped expression of eve, several other genes are expressed in a similar domain, including at least $o d d$, sob and $h h$. Based on their spatial relationships we suggest that these genes are activated by eve (either directly or through a close intermediary), but we cannot test this functionally since knocking down eve leads to a complete truncation of the growth zone and sequential segmentation does not take place. We do note that eve expression in the growth zone is not affected by knocking down odd or $h h$ (Additional file 2).

The next phase occurs in the anterior part of the anterior growth zone. Expression of a series of secondary pair-rule gene orthologs is activated, including at least opa and slp. We suggest that opa and $s l p$ are repressing each other, as there is no overlap in their expression domains at any point. Expression of $h h$ is maintained at this stage. Slightly anterior to where the secondary pair-rule genes are activated, expression of eve, odd and $s o b$ is switched off and the segment polarity genes inv and $w g$ start to be expressed in stripes. Interestingly, $h$, which is a pair-rule gene in Drosophila and in Tribolium [28], is expressed segmentally late in the cascade, anterior to the expression of $w g$ and inv, although it has an earlier non-segmental mesodermal expression. The point of activation of $i n v$ was previously defined as the border between the growth zone and the segmented germband [11]. Genes that are expressed in stripes at this border (the segment polarity genes and the secondary pair-rule genes) remain active throughout the germband stage and maintain their striped expression in each segment as the segments continue to mature.

Thus, segmentation from the growth zone occurs through three phases indicated by the expression of orthologs of primary pair-rule genes, secondary pair-rule genes and segment-polarity genes. The boundary between these phases is not sharp, and several genes are active across phases (e.g. hh). We have not looked at gap genes in the current work, since this group of genes and its role in segmentation has been studied previously [14-17]. In the Oncopeltus blastoderm, gap genes have a regulatory role in forming specific segments, as they do in Drosophila [4]. Existing data do not support a role for gap genes in the sequential segmentation cascade in Oncopeltus, since they are not expressed continuously in the growth zone, but only in nascent segments or at late stages of growth zone activity.

\section{Changes in the growth zone}

We have previously documented the changes in size of the growth zone throughout the segmentation process [11]. Here we expand on these results by documenting the expression levels of two genes holding central positions in the segmentation cascade, relative to the dynamic growth zone (Fig. 6d-e). Using the posteriormost blastoderm-derived segment - the T3 segment - as a fixed reference point, we show that the growth zone moves posteriorly as the germband elongates, and that nascent segments remain stationary at the point where they were first determined in the anterior growth zone. This leads us to the surprising conclusion that the early stripes of pair-rule gene expression already commit the cells where they are expressed to their future segmental identity. Cells expressing eve in the anterior growth zone remain in the same position as they go through the segmentation cascade, ultimately expressing inv as the anterior growth zone contracts latero-medially to give rise to a new segment of the germband.

In many species that have been studied, there is a phase wherein there is a wave of cyclical gene expression traveling across cells $[12,29,30]$. Our results are not consistent with a long-distance traveling wave in Oncopeltus. If there is a movement of expression across cells, it is only at the very early stage of eve expression, where a new stripe peels off of the posterior growth zone.

Our RNAi experiments raise an interesting contrast with mutant phenotypes of orthologous genes in Drosophila. None of our experiments result in the loss of specific segments or segmental domains. They all exhibit different levels of disruption of the segmental borders or segmental structure. This strengthens our assertion that segmental commitment occurs very early relative to that known from Drosophila. Indeed, knocking down eve, which we identify as the earliest gene in the cascade, leads to a complete loss of all growth zone derived segments. In addition, the weak RNAi phenotypes indicate a tightly integrated gene regulatory network, with a high level of redundancy.

\section{Conservation of the segment polarity network}

The segment polarity network is generally considered to be the most conserved part of the segmentation process in arthropods [31, 32]. This seems to hold for Oncopeltus. The expression border between $w g$ and inv/en defines the parasegment boundary in Drosophila [33]. We find the relative expression pattern of these genes is conserved in Oncopeltus. The position of inv relative to $\mathrm{wg}$ and $h h$ in the germband segments (Fig. 5) is deduced from the relative expression of eve and inv and by the co-expression of eve and $h h$ in the anterior growth zone (Fig. 4g), thus confirming that eve/inv, and $h h$ are expressed in the same part of the nascent segment, though not at the same time. Expression of $w g$ is adjacent and anterior to these, as in Drosophila.

The behavior of $h h$ is somewhat different from the two other segment polarity genes we studied, in that its expression begins much earlier in the cascade, concomitantly with the early expression of eve. This is similar to the early expression of $h h$ reported from the scorpion Euscorpius [34]. The early expression of $h h$ is an interesting indication of the processes of segment maturation. 
Given its position at the posterior border of the segment, the expression of $h h$ indicates that segment polarity and segment boundaries, usually perceived as late milestones in segment maturation, are actually established very early on, almost immediately as the tissue enters the anterior growth zone. Furthermore, when examining the progression of the expression pattern of $h h$ (Fig. $6 \mathrm{~d}$ ), we note that the posterior (early) $h h$ stripe is broad, not fully resolved and not separated from the second $h h$ stripe. Only the third or fourth stripe of $h h$, which coincides with the beginning of the expression of en/inv and $w g$, is completely resolved and with sharp borders. Similarly, in Drosophila, $h \mathrm{~h}$ is initially broadly expressed within the parasegmental unit, and is later refined to a narrower region. In addition to this, as demonstrated by double stainings, $h h$ is also expressed in the posterior growth zone, surrounded by a crescent of $w g$ expression. The relative expression of $h h$ and $w g$ in the posterior growth zone is the same as that later observed in the segment border.

\section{Relative rate of segmentation}

In previous work [11] we analyzed the dynamics of sequential segmentation from the growth zone, and showed that the rate of segment generation, using inv expression as a proxy, is not significantly different from linear throughout the process. In the present work, we were able to look at different phases of the segmentation process. Looking at the number of stripes of eve and other genes expressed in the anterior growth zone, we see that this number varies from 4 to 5 stripes in early stages to only 1 towards the end. We suggest that this indicates different processes that are not temporally linked. The first phase of segment determination, indicated by eve expression, occurs very rapidly, creating a "backlog" of segments waiting to go through the next phases, indicated by the expression of secondary pair-rule genes and segment polarity genes. Thus, by the time the first nascent abdominal segment starts expressing inv there are already 4-5 subsequent segments expressing eve. As segmentation progresses, primary determination slows down and final determination catches up, so that there is only one segment expressing eve.

\section{Evolution of the segmentation cascade}

Comparing our findings to what is known from better-studied experimental systems (most notably Drosophila and Tribolium) allows us to identify key aspects of the process that are broadly conserved at different phylogenetic scales and to reconstruct some of the evolutionary changes that have taken place in the evolution of the segmentation cascade, both within insects, and in arthropods more broadly.

The transcription factors commonly known as pair-rule genes hold a key early role that is conserved in the segmentation cascade of all arthropods studied to date. Although the gene studied most widely has been eve, orthologs of other members of this group interact with eve in many cases. These genes provide the first reiterated output that sets the path for segment determination. The signal driving pair-rule gene expression is variable and ranges from simultaneous activation in many segments as in Drosophila and in the Oncopeltus blastoderm [4], through Notch-signaling as in centipedes [26], to an endogenous pair-rule gene circuit as in Tribolium [21]. Nonetheless, the centrality of pair-rule genes in subsequent stages is conserved. In the case of Oncopeltus sequential segmentation, eve is very high in the cascade and is most likely upstream of all other segmentation genes.

Within pair-rule genes, a distinction between primary and secondary pair-rule genes is also broadly conserved, although the precise distinction of which genes fall into which category varies among taxa [35]. The primary pair-rule genes are active together at early phases of the cascade, while secondary pair-rule genes are active later, and are co-expressed with segment polarity genes further down the cascade.

\section{The evolution of double-segment patterning}

While we have been using the moniker "pair-rule genes", in reality the two-segment periodicity of these genes is probably taxonomically restricted to holometabolous insects. A two-segment periodicity is also found in geophilomorph centipedes [12], but this is likely to be independently evolved, since there is no evidence for such a periodicity in lithobiomorph centipedes [36], in chelicerates or in crustaceans. Double-segment patterning is common to most holometabolous insects, and we have previously suggested that it appeared at the base of Holometabola [4]. Within hemimetabolous insects, there is partial evidence for a pair-rule periodicity in segmentation in orthopterans [37, 38] where some of the eve stripes in the cricket Gryllus bimaculatus exhibit stripe splitting, whereas the gene pairberry exhibits splitting in the grasshopper Schistocerca americana. On the other hand, evidence from the cockroach Periplaneta americana [39] and the mayfly Ephoron leucon [40] (the former being closer to Paraneoptera and the latter being basal within winged insects [41]) indicate that segments are formed one by one, supporting single-segment periodicity as ancestral for insects. Regardless of whether the situation in Gryllus and Schistocerca represents a novelty for Orthoptera, or whether it is indicative of an earlier appearance of pair-rule periodicity, the single segment generation mode of Oncopeltus is probably representative of the ancestral single-segment situation from which pair-rule segmentation evolved.

The transition between single-segment patterning and double-segment patterning is significant. In geophilomorph centipedes this transition was probably accompanied by a 
doubling of segment number [42, 43]. In insects, there is no change in segment number, suggesting a very different mechanism. Double-segment patterning in insects requires each expression stripe of the pair-rule genes to translate to half a segment at later stages. Taking the Oncopeltus cascade as a hypothetical starting point, we can try to uncover the roots of the transition. The primary pair-rule genes are expressed in almost overlapping domains. However, the secondary pair-rule genes opa and $s l p$ are expressed in mutually exclusive domains, similar to those seen in double-segment patterning. We suggest that this mutually exclusive pattern within a single segment was elaborated to pattern consecutive segments, a process which also included a shift of the primary pair-rule gene expression domains to create non-overlapping sets of odd and even segment genes. Intriguingly, Oncopeltus does have one gene, encoding the nuclear receptor E75A, that is expressed in a double segment periodicity and shows a pair-rule phenotype upon being knocked down [19]. While we do not know how this gene fits into the segmentation cascade in Oncopeltus, it may indicate an early stage of segment identity definition, which was part of the basis for the evolution of a double-segment periodicity in patterning the segments.

The evolution of the double-segment patterning mode in holometabolous insects apparently involved several more fundamental differences in the way segments are patterned, relative to the putative ancestral mode seen in Oncopeltus. The definition of the segmental unit is much earlier in the Oncopeltus cascade, and is already manifested at the level of the primary pair-rule genes. The subsequent cascade refines the borders and possibly defines domains within the segment. Thus, when any gene in the cascade is knocked down (with the exception of eve), we see malformations in the segments and in their borders, but no loss of segments. Conversely, in holometabolous insects, knocking down genes higher than the segment-polarity level leads to loss of specific segments [2, 44-46].

\section{The evolution of simultaneous segmentation}

A key characteristic of the Drosophila segmentation cascade is the fact that segments are patterned simultaneously, as opposed to sequentially in the ancestral mode (often referred to as long germ vs. short germ development [47], respectively, but see Stahi and Chipman [4]). The transition between these two modes is not well understood. Two recent papers suggest, based on computational considerations, that the transition is actually fairly simple, and requires only minor changes in relative timing of the inputs to the cascade $[8,9]$. This is consistent with our observation that many aspects of the sequential segmentation cascade in Oncopletus are similar to the simultaneous cascade of Drosophila. However, both computational models assume input by gap genes, whereas we have no direct evidence of gap-gene input into the sequential cascade.

\section{Conclusions}

We have presented a detailed analysis of the genetic events involved in sequential segmentation in Oncopeltus. Coupled with our previous analysis of dynamic morphological events in this species, we provide a reference point for comparison with better studied species, to allow a reconstruction of the evolution of the segmentation process in insects. There is a remarkable degree of similarity among the studied insects at the level of molecular players and the general structure of the cascade, despite minor differences in the detail of network structure and significant differences in the cellular and morphological setting. Given the phylogenetic position of Oncopeltus relative to Tribolium and Drosophila, our analysis provides important insights into the evolution of one of the best studied developmental process - the generation of the segmented Drosophila blastoderm and sheds light on how developmental networks evolve.

\section{Methods}

\section{Embryo handling and staining}

All rearing, collecting, embryo fixing, staining and RNAi experiments, mounting and imaging were done using standard protocols, as described in previous work [11, $14,48]$. The main change is that for double staining we used Vector labs ImmPACT Vector Red (SK-5105) and Vector Blue (SK-5300) as the second AP substrates. Full protocols can be provided upon request.

\section{Gene cloning}

Standard PCR protocols and reagents were used in order to amplify gene fragments using Oncopeltus embryonic cDNA as a template. Clone identity was verified via sequencing and aligning with the annotated genes of the Oncopeltus genome.

eve, cad, $D l$ and inv fragments used in this paper are the same as in [32].

$w g$ was cloned in our lab, on the basis of [22]. GenBank accession number AY899335.1.

GenBank accession numbers and primer sequences for the rest of the genes cloned for this paper are listed in Additional File 3.

\section{RNAi experiments}

For each of the genes, dsRNA was synthesized in a single, 3-h transcription reaction (total volume $25 \mu \mathrm{l}$ ) with a MEGAscript T7 kit (AM1334, Invitrogen). The transcription reaction was terminated using a standard ethanol precipitation protocol. The resulting dsRNAs were then suspended in 10x isotonic injection buffer $(5 \mathrm{mM}$ $\mathrm{KCl}, 0.1 \mathrm{mM} \mathrm{NaH2PO} 4)$ so that final concentration of 
the injected dsRNA was $0.2-3 \mu \mathrm{g} / \mu \mathrm{l}$. For each gene, we generated two non-overlapping (or minimally overlapping) fragments. Primer sequences are listed in Additional File 3.

For each experiment 4 females were isolated during the 5th instar to make sure treated females are virgins (as this significantly improves the dsRNA penetrance). Injection of the dsRNA was then performed between 2 to 4 days after female larvae molted to adults. In order to perform the injection, virgin adult females were first anaesthetized by putting them in a closed box and pumping in $\mathrm{CO} 2$ gas until movement stopped. Injection and egg collection were done as previously reported [4]. For most clutches, we allowed four eggs to develop to hatching or close to hatching to assess larval phenotypes, and fixed the other eggs at different times to analyze the effects of RNAi on development. For each gene, the second fragment was injected to a smaller number of females and only a few clutches were collected, in order to confirm that the larval phenotypes were similar to those obtained by experiments with the first fragment.

\section{Microscopy and imaging}

Images of hatchlings were captured using a Nikon 'digital sight' console connected to a DS-Fi1 digital camera mounted on either a SMZ1500 Nikon dissecting scope or an AZ100 Zoom Stereoscope.

Images of slide-mounted embryos were captured with the same console and camera mounted on an Eclipse 80i Nikon Microscope.

\section{Gene expression intensity measurements (Fig. 6)}

Semi-quantitative gene expression intensity measurements were done by measuring the gray-scale intensity of the region of interest in captured images of Of- $h h$ and Of-eve stained embryos. This was done using the ROI manager of the FIJI software package [49] (analyze $>$ tools $>$ ROI manager, followed by more $>$ multi measure $>$ list $>$ save as, to add measurements to a list).

Images were all taken with the same magnification $(x$ 10). With the FIJI 'stack' option, images of Of- $h h$ and Of-eve stained embryos were stacked separately for easier data collection and image processing. Images were individually aligned horizontally for easier intensity measurements using a rectangle selection tool. Image colors were inverted in order to represent the gene expressing areas as bright areas, (presented in the data and graphs as higher expression) and then measured individually using the FIJI ROI manger. Distorted embryos were excluded from the sample.

In order to prevent bias, the area measured was the entire width of the embryo, and included the entire length of the abdomen, recognized by Of- $h h$ expression at the T3 border or by morphological identification of T3 in the Of-eve staining.

Measurement output was one csv file containing the intensity measurements of the entire stack of embryos (see Additional files 4, 5 for raw data). Under the assumption that the length of the abdomen represents a good approximation of developmental stage, we sorted the data according to the total number of data points of each column, each point representing a pixel in the original image, hence sorting the embryos roughly according to developmental stage. This assumption was proven to be robust, as the segmental stripes aligned almost perfectly, as seen in the Of- $h h$ stain. This validation enabled us to proceed and use the same method with the Of-eve stained embryos.

When necessary, deletion of first few data points was done when data points were clearly seen to be taken from the background.

Normalization of the intensity measurements for Of-eve were done separately for each embryo, by dividing all the values by the strongest value, hence setting the strongest intensity to one. Of- $h h$ measurements were all normalized to the weakest expression point found in all of the Of- $h h$ intensity data, mainly for aesthetic reasons.

The length of the embryos in pixels is indicated on the length axis of Fig. 6., and spans between 470 (shortest, youngest) and 870 (longest, older embryos) pixels. By close approximation, each pixel equals $1 \mu \mathrm{m}$ (Calibrated manually).

3D Graphs (x,y,z plots) were produced using the Plotly $\mathrm{R}$ package (Plotly Technologies Inc..)

\section{Additional files}

Additional file 1: Additional examples of embryonic and hatchling phenotype of the Of-slp, Of-hh and Of-odd RNAi experiments. All embryos are stained for the inv segmental marker. Younger embryos are in the left column, older embryos on the middle column, hatchlings on the right column. Embryos stages are not identical in all columns for all experiments, specimens in the same row are not linked. (PDF 14900 kb)

Additional file 2: eve staining of RNAi embryos. In addition to inv staining shown in the main text, eve staining was also performed on RNAi embryos. In relation to early and mid-stage WT embryos ( $\left.A, A^{\prime}\right)$ no substantial aberration of eve expression is seen in the GZ for any of the RNAi experiments. (B) in odd-RNAi embryos, eve is expressed ectopically in several medial patches in the thoracic and gnathal segments (arrow and arrowhead). When compared to the embryo in Fig. 7b, it seems that these points correspond to the areas where the thoracic segments fuse, or possibly to areas in the inv stripe that is more weakly expressed. (C) eve expression in s/p-RNAi embryos is slightly out of the norm for the stage of the embryo, but this is mostly due to the abnormal general shape of the embryo. (D) hh-RNAi embryo displaying ectopic eve expression in the head, corresponding to the head phenotype displayed in Fig. 7d-d". (PDF 1910 kb)

Additional file 3: Primers. Primers used to clone fragments for RNA in situ hybridization (upper table) and to clone dsRNA for RNAi experiments (lower table). (DOCX $14 \mathrm{~kb}$ )

Additional file 4: Raw measurement data for $h h$. CSV file including the raw measurement data for hh expression. Each column is a single 
embryo, with each cell representing a summation of pixel intensity across the embryo at a single position along the A-P axis. (CSV $457 \mathrm{~kb}$ )

Additional file 5: Raw measurement data for eve. CSV file including the raw measurement data for eve expression. Each column is a single embryo, with each cell representing a summation of pixel intensity across the embryo at a single position along the A-P axis. (CSV $530 \mathrm{~kb}$ )

\section{Abbreviations}

DAPI: 4',6-diamidino-2-phenylindole; dsRNA: Double stranded ribonucleic acid; RNAi: Ribonucleic acid interference; T3: Third thoracic segment

\section{Acknowledgments}

We thank Oren Lev and Netta Kasher for help in preparing Figs. 5 and 6.

\section{Funding}

This work was funded by a grant from the NSF / BSF IOS program: BSF 2012763, and by an Israel Science Foundation grant \#120/16.

\section{Availability of data and materials}

All data are included within the manuscript and Additional Material. New gene sequences have been deposited in NCBI as detailed in the Additional Material. All clones can be provided by the authors upon request.

\section{Authors' contributions}

TA designed and carried out all experiments, analyzed the data and wrote a draft of the paper. ADC supervised the work, participated in the analysis and wrote the final version of the paper. Both authors read and approved the final version.

\section{Ethics approval and consent to participate}

All work reported herein was on invertebrate animals that do not require ethical approval.

\section{Consent for publication}

N/A

\section{Competing interests}

The authors declare they have no competing interests.

\section{Publisher's Note}

Springer Nature remains neutral with regard to jurisdictional claims in published maps and institutional affiliations.

Received: 10 June 2018 Accepted: 12 November 2018 Published online: 28 November 2018

\section{References}

1. Peel AD, Chipman AD, Akam M. Arthropod segmentation: beyond the Drosophila paradigm. Nat Rev Genet. 2005;6(12):905-16.

2. Lawrence PA. The making of a fly: the genetics of animal design. Oxford: Blackwell Scientific Publications; 1992.

3. Hartenstein V, Chipman AD. Hexapoda: a Drosophila's view of insect development. In: Wanninger A, editor. Evolutionary Developmental Biology of Invertebrates, vol. 5. Vienna: Springer; 2015. p. 1-91.

4. Stahi R, Chipman AD. Blastoderm segmentation in Oncopeltus fasciatus and the evolution of arthropod segmentation mechanisms. Proc R Soc Lond B. 2016;283:20161745.

5. Peel A. The evolution of arthropod segmentation mechanisms. Bioessays. 2004:26(10):1108-16.

6. Jaeger J, Surkova S, Blagov M, Janssens H, Kosman D, Kozlov KN, Manu ME, Vanario-Alonso CE, Samsonova M, et al. Dynamic control of positional information in the early Drosophila embryo. Nature. 2004;430(6997):368-71.

7. Verd B, Clark E, Wotton KR, Janssens H, Jimenez-Guri E, Crombach A, Jaeger J. A damped oscillator imposes temporal order on posterior gap gene expression in Drosophila. PLoS Biol. 2018;16(2):e2003174.

8. Clark E. Dynamic patterning by the Drosophila pair-rule network reconciles long-germ and short-germ segmentation. PLoS Biol. 2017;15(9):e2002439.
9. Zhu X, Rudolf H, Healey L, François P, Brown SJ, Klingler M, El-Sherif E. Speed regulation of genetic cascades allows for evolvability in the body plan specification of insects. Proc Natl Acad Sci. 2017;114:E8646-55.

10. Chipman AD. Oncopeltus fasciatus as an evo-devo research organism. Genesis. 2017;55:e23020.

11. Auman T, Vreede BMI, Weiss A, Hester SD, Williams TA, Nagy LM, Chipman AD. Dynamics of growth zone patterning in the milkweed bug Oncopeltus fasciatus. Development. 2017;144:1896-905.

12. Chipman AD, Arthur W, Akam M. A double segment periodicity underlies segment generation in centipede development. Curr Biol. 2004;14:1250-5.

13. Macdonald PM, Ingham P, Struhl G. Isolation, structure, and expression of even-skipped: a second pair-rule gene of Drosophila containing a homeo box. Cell. 1986;47(5):721-34.

14. Ben-David J, Chipman AD. Mutual regulatory interactions of the trunk gap genes during blastoderm patterning in the hemipteran Oncopeltus fasciatus. Dev Biol. 2010;346:140-9.

15. Liu PZ, Kaufman TC. Krüppel is a gap gene in the intermediate germband insect Oncopeltus fasciatus and is required for development of both blastoderm and germband-derived segments. Development. 2004;131(18):4567-79.

16. Liu PZ, Kaufman TC. hunchback is required for suppression of abdominal identity, and for proper germband growth and segmentation in the intermediate germband insect Oncopeltus fasciatus. Development. 2004;131(7):1515-27.

17. Liu PZ, Patel NH. giant is a bona fide gap gene in the intermediate germband insect, Oncopeltus fasciatus. Development. 2010;137(5):835-44.

18. Ginzburg N, Cohen M, Chipman AD. Factors involved in early polarization of the anterior-posterior axis in the milkweed bug Oncopeltus fasciatus. Genesis. 2017;55:e23027.

19. Erezyilmaz DF, Kelstrup HC, Riddiford LM. The nuclear receptor E75A has a novel pair-rule-like function in patterning the milkweed bug, Oncopeltus fasciatus. Dev Biol. 2009:334(1):300-10.

20. Liu PZ, Kaufman TC. even-skipped is not a pair-rule gene but has segmental and gap-like functions in Oncopeltus fasciatus. an intermediate germband insect Development. 2005;132(9):2081-92.

21. Choe CP, Miller SC, Brown SJ. A pair-rule gene circuit defines segments sequentially in the short-germ insect Tribolium castaneum. Proc Natl Acad Sci U S A. 2006;103(17):6560-4.

22. Angelini DR, Kaufman TC. Functional analyses in the milkweed bug Oncopeltus fasciatus (Hemiptera) support a role for Wnt signaling in body segmentation but not appendage development. Dev Biol. 2005;283(2):409-23.

23. Williams TA, Nagy LM. Linking gene regulation to cell behaviors in the posterior growth zone of sequentially segmenting arthropods. Arthropod Struct Dev. 2017:46:380-94.

24. Stollewerk A, Schoppmeier M, Damen WGM. Involvement of Notch and Delta genes in spider segmentation. Nature. 2003;423(6942):863-5.

25. Schoppmeier M, Damen WGM. Suppressor of hairless and Presenilin phenotypes imply involvement of canonical notch-signalling in segmentation of the spider Cupiennius salei. Dev Biol. 2005;280(1):211-24.

26. Chipman AD, Akam M. The segmentation cascade in the centipede Strigamia maritima: involvement of the notch pathway and pair-rule gene homologues. Dev Biol. 2008;319(1):160-9.

27. Williams T, Blachuta B, Hegna TA, Nagy LM. Decoupling elongation and segmentation: notch involvement in anostracan crustacean segmentation. Evol Dev. 2012:14(4):372-82.

28. Aranda M, Marques-Souza H, Bayer T, Tautz D. The role of the segmentation gene hairy in Tribolium. Dev Genes Evol. 2008;218(9):465-77.

29. El-Sherif E, Averof M, Brown SJ. A segmentation clock operating in blastoderm and germband stages of Tribolium development. Development. 2012;139(23):4341-6.

30. Eriksson BJ, Ungerer $P$, Stollewerk A. The function of notch signalling in segment formation in the crustacean Daphnia magna (Branchiopoda). Dev Biol. 2013;383(2):321-30.

31. von Dassow G, Meir E, Munro EM, Odell GM. The segment polarity network is a robust developmental module. Nature. 2000;406:188-92.

32. Auman T, Chipman AD. The evolution of gene regulatory networks that define arthropod body plans. Int Comp Biol. 2017;57(3):523-32.

33. Martinez-Arias A, Lawrence PA. Parasegments and compartments in the Drosophila embryo. Nature. 1985;313:639-42.

34. Simonnet F, Deutsch J, Queinnec E. Hedgehog is a segment polarity gene in a crustacean and a chelicerate. Dev Genes Evol. 2004;214(11):537-45.

35. Chipman AD. Thoughts and speculations on the ancestral arthropod segmentation pathway. In: Minelli A, Fusco G, editors. Evolving pathways: 
key themes in evolutionary developmental biology. Cambridge: Cambridge University Press; 2008. p. 339-55.

36. Hughes CL, Kaufman TC. Exploring myriapod segmentation: the expression patterns of even-skipped, engrailed, and wingless in a centipede. Dev Biol. 2002;247:47-61.

37. Mito T, Kobayashi C, Sarashina I, Zhang HJ, Shinahara W, Miyawaki K, Shinmyo Y, Ohuchi H, Noji S. even-skipped has gap-like, pair-rule-like, and segmental functions in the cricket Gryllus bimaculatus, a basal, intermediate germ insect (Orthoptera). Dev Biol. 2007;303(1):202-13.

38. Davis GK, Jaramillo CA, Patel NH. Pax group III genes and the evolution of insect pair-rule patterning. Development. 2001;128(18):3445-58.

39. Chesebro J. Mechanisms of segmentation in the American cockroach, Periplaneta americana. University of Sussex: Unpublished Ph.D thesis; 2012.

40. O'Donnell BC, Jockusch EL. The expression of wingless and Engrailed in developing embryos of the mayfly Ephoron leukon (Ephemeroptera: Polymitarcyidae). Dev Genes Evol. 2010;220(1-2):11-24.

41. Misof B, Liu SL, Meusemann K, Peters RS, Donath A, Mayer C, Frandsen PB, Ware J, Flouri T, Beutel RG, et al. Phylogenomics resolves the timing and pattern of insect evolution. Science. 2014;346(6210):763-7.

42. Bonato L, Foddai D, Minelli A. Increase by duplication and loss of invariance of segment number in the centipede Mecistocephalus microporus (Chilopoda, Geophilomorpha, Mecistocephalidae). Ital J Zool. 2001:68(4):345-52.

43. Minelli A, Chagas-Junior A, Edgecombe GD. Saltational evolution of trunk segment number in centipedes. Evol Dev. 2009;11(3):318-22.

44. Choe CP, Brown SJ. Evolutionary flexibility of pair-rule patterning revealed by functional analysis of secondary pair-rule genes, paired and sloppy-paired in the short-germ insect, Tribolium castaneum. Dev Biol. 2006;302:281-94.

45. Xiang J, Reding K, Heffer A, Pick L. Conservation and variation in pair-rule gene expression and function in the intermediate-germ beetle Dermestes maculatus. Development. 2017;144(24):4625-36.

46. Nüsslein-Volhard C, Wieschaus E. Mutations affecting segment number and polarity in Drosophila. Nature. 1980;287(5785):795-801.

47. Davis GK, Patel NH. Short, long, and beyond: molecular and embryological approaches to insect segmentation. Ann Rev Entom. 2002;47:669-99.

48. Birkan M, Schaeper ND, Chipman AD. Early patterning and blastodermal fate map of the head in the milkweed bug Oncopeltus fasciatus. Evol Dev. 2011;13(5):436-47.

49. Schindelin J, Arganda-Carreras I, Frise E, Kaynig V, Longair M, Pietzsch T, Preibisch S, Rueden C, Saalfeld S, Schmid B, et al. Fiji: an open-source platform for biological-image analysis. Nat Methods. 2012;9(7):676-82.

Ready to submit your research? Choose BMC and benefit from:

- fast, convenient online submission

- thorough peer review by experienced researchers in your field

- rapid publication on acceptance

- support for research data, including large and complex data types

- gold Open Access which fosters wider collaboration and increased citations

- maximum visibility for your research: over $100 \mathrm{M}$ website views per year

At $\mathrm{BMC}$, research is always in progress.

Learn more biomedcentral.com/submissions 